Research Article

\title{
Research on the Normal Use of a Plug Discharge Tunnel
}

\author{
Wen Liu, Jun Deng ${ }^{(D}$, Zhong Tian, and Faxing Zhang \\ State Key Laboratory of Hydraulics and Mountain River Engineering, Sichuan University, Chengdu 610065, China \\ Correspondence should be addressed to Jun Deng; djhao2002@scu.edu.cn
}

Received 23 September 2019; Revised 28 November 2019; Accepted 13 January 2020; Published 12 February 2020

Academic Editor: Vincenzo Vespri

Copyright $(92020$ Wen Liu et al. This is an open access article distributed under the Creative Commons Attribution License, which permits unrestricted use, distribution, and reproduction in any medium, provided the original work is properly cited.

\begin{abstract}
This study analyzed the normal use of an unusual flood-releasing tunnel with a plug dissipator. Firstly, normal physical model tests based on the Froude criterion $(1: 50)$ were finished. Secondly, depression physical model tests based on the Froude criterion (1: 50) and cavitation similarity criterion were finished. Thirdly, 3-dimensional numerical simulation of flow field was finished, and free surface profile was captured, which was based on RNG $k-\varepsilon$ two-equation turbulence model and VOF method. The focus of this study is on the relationship between normal use and cavitation characteristics (e.g., pressure, turbulence kinetic energy, and cavitation number). The results show that lowering the reservoir water level, reduced by $20.41 \mathrm{~m}$ at most, increases the risk of cavitation of a plug discharge tunnel, which means with the decrease of the flow cavitation number, the possibility of structural damage will increase dramatically, while reducing the outlet height can effectively raise the flow cavitation number, ensuring the safety of normal use. Under the conditions of free outflow, for the $H_{1} / e$ values of $4.45,4.00$, and 3.55, the conditions in which the tunnel meets the requirements of anticavitation are $h / D \leq 0.42, h / D \leq 0.39$, and $h / D \leq 0.35$, respectively. In addition, the discharge capacity of the tunnel is not significantly reduced with the lowering of outlet height, implying that operation under a low water head of the plug discharge tunnel, as low as 3.55 of $H_{1} / e$ in the test, is feasible. The results obtained in this study can serve as reference information in engineering design of the plug discharge tunnel.
\end{abstract}

\section{Introduction}

Rebuilding the diversion tunnel into the discharge tunnel is a cost-effective approach that can reasonably utilize waste resources and increase the operational flexibility of engineering operations. The plug is a new internal energy dissipator that realizes the goal of drastic energy dissipation through the sudden enlargement and contraction of the water-crossing section [1]. Unlike the orifice energy dissipator, the plug energy dissipator overcomes the drawback of easy cavitation at a sharp-edged orifice, enabling it to obtain an outstanding performance with regard to structural strength, energy dissipation effect, and antiresistance [2, 3].

Flow recovering length is an important concern because it determines the distance of adjacent multistage plugs. Only when the plug spacing is greater than the flow recovering length can the energy dissipation rate be maximum. Many researchers have studied the shape design of the plug dissipator. Ai and Ding [4] researched reasonable distance between the upper and the lower plug in multistage plug discharge tunnel. Ai and Ding [5] also studied the effect of contraction ratio to the minimum wall pressure coefficient with RNG $k-\varepsilon$ two-equation turbulence model and proposed a quantitative calculation formula. Li et al. [6] studied the way of adding vent holes in the recovering area of the plug to increase the possibility of anticavitation damage of the plug flood tunnel and obtained positive results. The results show that, by setting vent holes in the recovering area, air can be diffused to the two side walls, thereby effectively protecting the plug discharge tunnel. These studies mainly focused on the influence of different plug geometries on the flow characteristics, such as wall pressure characteristics and aeration protection length.

At present, many hydraulic projects have adopted plug energy dissipator. The temporary weep hole of the Glen Canyon dam in the USA and the bottom outlet of the Mica dam [7] in Canada have both adopted the plug energy dissipator. The Mica dam rebuilt a diversion tunnel with a water head of $180 \mathrm{~m}$ into a permanent bottom outlet. Although the discharge is not large, its successful use has 
played a strong role in promoting the engineering application of the plug. In China, the first use of an orifice dissipator and its operational implementation was in the Xiaolangdi Project located in the Yellow River, which underwent a prototype test under low-water lever conditions with no observed cavitation $[8,9]$. However, strong cavitation noise was subsequently observed during normal operation. The Xiluodu Project located in the Jinsha River studied the flood plugging tunnel of the pressurized shaft, and it was found that its energy dissipation rate and anticavitation performance still meet the requirements under a flow rate as high as $2700 \mathrm{~m}^{3} / \mathrm{s}$, indicating that the use of a plug energy dissipator for releasing larger discharge is feasible [10]. The Houziyan Hydropower Station located in the Dadu River used a multistage plug to transform the diversion tunnel into a flood discharge tunnel $[11,12]$. The atmospheric pressure test, numerical simulations, and decompression test together showed that the structure satisfies the anticavitation requirement, and the energy dissipation rate of the entire tunnel was also improved to a relatively high level. These engineering experiences show that plug dissipator has the advantages of high energy dissipation rate, safety, and simple structure geometry.

However, in view of the fact that the plug is still a new type of energy dissipator, it is often designed as an unusual flood discharge tunnel in engineering, which means it is only used in operation under extreme conditions. This causes the resources to be wasted and is not conducive to engineering practice and promotion of the plug energy dissipation. Since the plug energy dissipator must have sufficient wall pressure to avoid cavitation damage [13, 14], the tunnel outlet elevation is generally designed to be relatively low so that the outlet could be submerged under the downstream water level, and the reservoir water level must be sufficiently high to ensure high pressure in the tunnel. Operation of the tunnel at a low water level may reduce the wall pressure or even give rise to cavitation. According to the analysis, reducing the height of the outlet can increase the pressure inside the tunnel and enhance the antiresistance of the flood discharge tunnel so that even relatively low upstream and downstream water levels can ensure the safety of the flood discharge of the tunnel. However, the decrease in the outlet height will affect the discharge capacity and energy dissipation rate of the tunnel. How to solve the contradiction between the normal operational safety and discharge capacity and the energy dissipation rate of the plug discharge tunnel is a problem worth exploring.

This paper attempts to answer this question. The numerical model is developed due to factors such as economy, portability, and time-saving by comparing it with physical experiments. The numerical model is very flexible for adjusting parametric parameters and provides complete information of flow field, such as velocity profile and pressure field. The main focus of the present study is to investigate the features of discharge capacity, pressure, energy dissipation rate, and cavitation number of a plug discharge tunnel. This work can provide references to prevent or reduce the risk of cavitation in the plug discharge tunnel in hydraulic engineering.

\section{Physical Structure of the Plug Discharge Tunnel}

The object of this study is the plug type discharge tunnel of Houziyan Hydropower Station in China. The design of the plug discharge tunnel is shown in Figure 1. The dimensions of the inlet gate are $9 \mathrm{~m} \times 11 \mathrm{~m}$ (width $\times$ height), and the altitude difference between the inlet and outlet floor is $93 \mathrm{~m}$. The entire flood discharge energy dissipation system consists of 6 plugs. Two vertical plugs (plugs 1 and 2) with the heights of $15.0 \mathrm{~m}$ and $6.0 \mathrm{~m}$, respectively, are arranged in the shaft section, the outlet diameters are $7.0 \mathrm{~m}$ and $8.5 \mathrm{~m}$, respectively, and the spacing between the two vertical plugs is $24 \mathrm{~m}$. The second vertical plug is connected to the top of a rectangular baffle chamber, and the dimensions of the chamber are $13.0 \mathrm{~m} \times 15.0 \mathrm{~m} \times 30.0 \mathrm{~m}$ (width $\times$ height $\times$ length). After the baffle chamber, a slope plug is placed (plug 3 ) with the length of $25.0 \mathrm{~m}$ and the outlet dimensions of $10.0 \mathrm{~m} \times 4.5 \mathrm{~m}$ (width $\times$ height). Three horizontal plugs (plugs 4,5 , and 6 ) are located after the slope plug. The inlet diameter of all three plugs is $12.0 \mathrm{~m}$, and their outlet diameters are $9.0 \mathrm{~m}, 9.2 \mathrm{~m}$, and $9.8 \mathrm{~m}$, respectively. The distance between plugs 3 and 4 is $60.0 \mathrm{~m}$, and the spacing between the three horizontal plugs are both $40.0 \mathrm{~m}$. The lower horizontal section is in the form of an arched section, with a straight wall height of $11.5 \mathrm{~m}$ and a top arch radius of $7.8 \mathrm{~m}$. The outlet of the tunnel has a "curve pressure slope outlet" body shape, and the width of the pressure slope plane gradually narrows from $13.0 \mathrm{~m}$ to $11.0 \mathrm{~m}$. The "curve pressure slope outlet" body shape is made up of a circular curve, which requires the horizontal distance limited to be $26.1 \mathrm{~m}$, and the tangent line of curve end needs to be horizontal. The variation in the height $h$ of the pressure outlet is $4.0 \sim 7.5 \mathrm{~m}$, and the top plate is connected by a single arc curve. The horizontal length of the curve is kept unchanged at $26.1 \mathrm{~m}$, and the radius ensures that the angle between the endpoint tangent and the horizontal direction is zero.

\section{Investigation Methods}

3.1. Normal Test of Physical Model Experiments. The scale of the normal model is $1: 50$ based on the Froude criterion for experimental investigations (Figure 2). The experimental operational conditions are shown in Table 1 . The test numbers 1-8 in Table 1 are unusual operational conditions in which the upstream and downstream water levels are unchanged $\left(H_{1} / e=5.40\right)$, and the outlet submergence degree $H_{2} / h$ increases from 2.76 to 5.18 with the decrease in the outlet height. The normal operational conditions 9-32 consider the downstream as the limiting condition, which means that the outlet is always free discharge. In the table, $H_{1}$ is the upstream hydraulic head of $49 \mathrm{~m}, 44 \mathrm{~m}$, and $39 \mathrm{~m}$, $e$ is the height of gate, and $\mathrm{H}_{2}$ is the downstream hydraulic head (test numbers $1-8$ are $20.7 \mathrm{~m}$, and test numbers 9-32 are equivalent to $h$, changing from $4.0 \mathrm{~m}$ to $7.5 \mathrm{~m}$ ). The hydraulic diameter $D$ of the lower horizontal section, as a constant, is $14.13 \mathrm{~m}$, and $D$ is defined as $4 A_{0} / C$, in which $A_{0}$ $\left(A_{0}=181.53 \mathrm{~m}^{2}\right)$ and $C\left(C=51.38 \mathrm{~m}^{2}\right)$ are the cross-sectional area and perimeter of a lower horizontal section, respectively. 


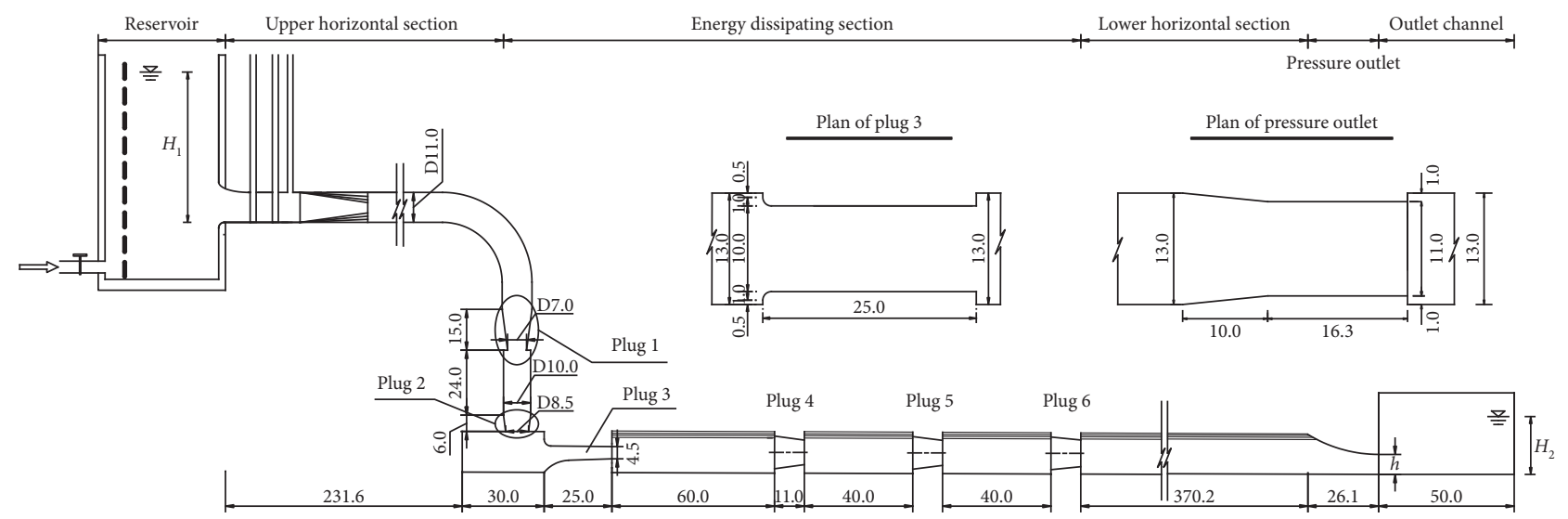

Figure 1: Physical dimensions of the plug discharge tunnel (unit is $\mathrm{m}$ ).

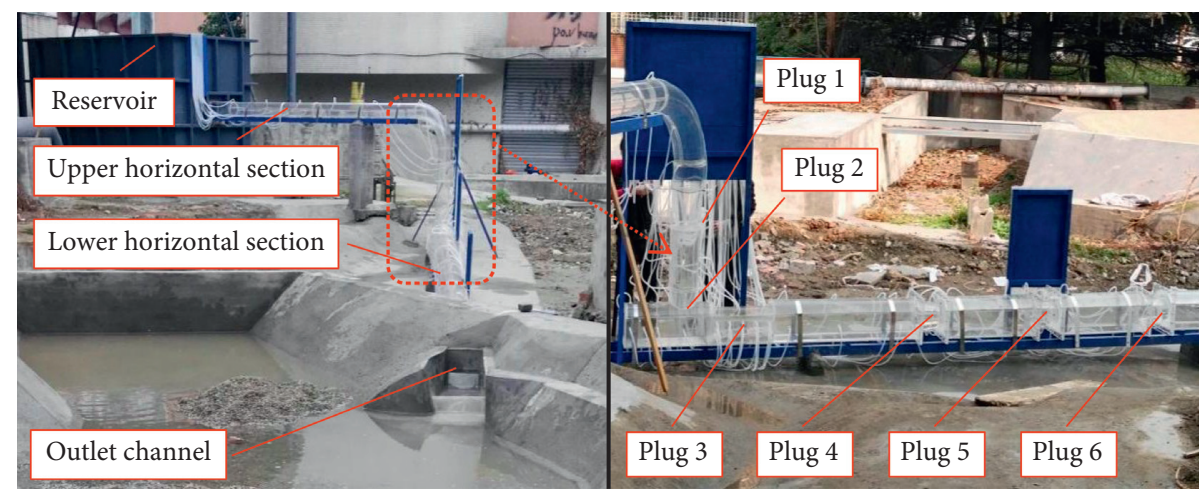

FIgURE 2: The physical model layout.

TABLE 1: Experimental operational conditions.

\begin{tabular}{lcccc}
\hline Test number & $H_{1} / e$ & $H_{2} / h$ & $h / D$ & Comments \\
\hline 1 & 5.40 & 2.76 & 0.53 & Normal test, depression test, numerical test \\
2 & 5.40 & 2.96 & 0.50 & Numerical test \\
3 & 5.40 & 3.18 & 0.46 & Numerical test \\
4 & 5.40 & 3.45 & 0.42 & Normal test, numerical test \\
5 & 5.40 & 3.76 & 0.39 & Numerical test \\
6 & 5.40 & 4.14 & 0.35 & Normal test, numerical test \\
7 & 5.40 & 4.60 & 0.32 & Numerical test \\
8 & 5.40 & 5.18 & 0.28 & Numerical test \\
$9 \sim 32$ & $4.45,4.00,3.55$ & Free outflow & $0.53 \sim 0.28$ & Numerical test \\
\hline
\end{tabular}

3.2. Depression Test of Physical Model Experiments. The scale of the depression test model is $1: 50$, which is based on the Froude criterion and the cavitation similarity criterion for experimental investigations. All the shape parameters are just the same with normal test above. The time-averaged cavitation number [15] can be defined as

$$
\sigma=\frac{\left(\left(p_{u} / \gamma\right)+\left(p_{a} / \gamma\right)-\left(p_{v} / \gamma\right)\right)}{\left(v^{2} /(2 g)\right)}
$$

where $p_{u} / \gamma$ and $v^{2} /(2 g)$ are the pressure head and velocity head of each section, respectively. $p_{a}$ is the atmospheric pressure, and $p_{v}$ is the saturated vapor pressure. $g$ is the gravitational acceleration, $9.81 \mathrm{~m} / \mathrm{s}^{2} . \gamma$ is the unit weight, $9810 \mathrm{~N} / \mathrm{m}^{3} . v$ is the velocity.

To ensure safety, the local atmospheric pressure shall be the atmospheric pressure corresponding to the elevation at which the upper horizontal section is located [15]:

$$
\frac{p_{a}}{\gamma}=10.33-\frac{1786}{900}=8.85 \mathrm{~m},
$$

where $p_{v} / \gamma$ is quite sensitive to temperature. The prototype value adopts the water temperature of $20^{\circ} \mathrm{C}$, that is, $0.24 \mathrm{~m}$ water column pressure. 
Under similar conditions, the cavitation number in the decompression test should be equal to the cavitation number in prototype, that is [15],

$$
\left(\frac{\left(p_{u} / \gamma\right)+\left(p_{a} / \gamma\right)-\left(p_{v} / \gamma\right)}{\left(v^{2} /(2 g)\right)}\right)_{m}=\left(\frac{\left(p_{u} / \gamma\right)+\left(p_{a} / \gamma\right)-\left(p_{v} / \gamma\right)}{\left(v^{2} /(2 g)\right)}\right)_{p}
$$

The subscript $m$ (model) in the formula represents the relevant hydraulic parameters in the decompression test, and the subscript $p$ (prototype) represents the relevant hydraulic parameters in the prototype.

Therefore, under the premise of gravity similarity criterion, if the cavitation number is similar, the atmospheric pressure in the depression tank should be as follows [15]:

$$
\frac{p_{a m}}{\gamma}=\frac{\left(p_{a p} / \gamma\right)-\left(p_{v p} / \gamma\right)}{\lambda_{l}}+\frac{p_{v m}}{\gamma}=\frac{8.85-0.24}{50}+0.24=0.42 \mathrm{~m}
$$

The vacuum degree to be controlled in the depression tank is as follows [15]:

$$
\frac{p_{0 m}}{\gamma}=\frac{p_{a l}}{\gamma}-\frac{p_{a m}}{\gamma}=9.81-0.42=9.39 \mathrm{~m},
$$

where $p_{a l} / \gamma$ is the atmospheric pressure at the location of the depression tank during the test and $p_{a m} / \gamma$ is the vacuum pressure to be controlled in the depression tank under similar conditions.

Relative vacuum degree can be expressed as follows [15]:

$$
\eta_{m}=\frac{p_{0 m} / \gamma}{p_{a l} / \gamma}=\frac{9.39}{9.81}=95.7 \%
$$

The maximum relative vacuum degree of the depression equipment in the laboratory is close to $99 \%$. In addition to satisfying similar condition, there is still considerable surplus. If no cavitation occurs, we will continue to improve the relative vacuum degree until we find the incipient cavitation number. The related depression test equipment is shown in Figure 3.

The outlet height of $7.5 \mathrm{~m}$ is verified by the $1: 50$ decompression test, and the sound pressure signals are shown in Figure 4. In the effective analysis frequency range of $35 \sim 160 \mathrm{kHz}$, the maximum sound pressure difference of the section of plugs 3-6 under similar vacuum pressure does not exceed $5 \mathrm{~dB}$. However, experimentally, an occasional weak cavitation was observed at the exit of plugs 5 and 6 . Although the cavitation was not collapsed in the sidewall, the situation was almost critical, and if it was considered for normal use, it is very likely that cavitation damage would occur. When vacuuming to $98.5 \%$ by the decompression test, the water flow noise exceeds the environmental noise by $5 \mathrm{~dB}$, and the cavitation number $\sigma$ at this time was used as the incipient cavitation number $\sigma_{i}$ of the different parts of the plug discharge tunnel. The incipient cavitation numbers of $X=220 \mathrm{~m}$,
$X=280 \mathrm{~m}, X=300 \mathrm{~m}$, and $X=480 \mathrm{~m}$ are $15.73,11.40,8.51$, and 6.61 , respectively.

Note that the reason for the choice of effective analysis frequency range of $35 \sim 160 \mathrm{kHz}$ is that when the frequency is lower than $35 \mathrm{kHz}$, the experimental results are greatly affected by the ambient noise, while cavitation usually occurs at high frequency, which means low frequency signals do not have a substantial effect on experimental results. In addition, $160 \mathrm{kHz}$ is the maximum sampling frequency of the hydrophone.

3.3. Numerical Simulations. Since the research object is a closed pressurized structure, the conventional physical model test cannot effectively measure the internal characteristics of flow field, and numerical simulations provide a good solution for this problem. Considering that the sudden expansion and contraction of the plug will cause a sharp change in the streamline, the three-dimensional RNG $k$ - $\varepsilon$ two equation turbulence model was used to simulate the turbulent flow [16, 17]. The control volume method was introduced to discretize governing equations. The SIMPLER method [18], which has good convergence characteristics, was used for pressure-velocity coupling [19]. The no-slip boundary condition was used on the wall [20]. The standard wall function was chosen to specify the near wall regions of the flow. Experience shows that, for reinforced concrete, roughness height, $K_{s}$, equal to $0.0025 \mathrm{~m}$ is relatively appropriate. And this research considers that the roughness on the concrete surface is evenly distributed, so the value of roughness constant, $C_{s}$, is equal to 0.5 . The governing equations are follows [21].

Continuity equation:

$$
\frac{\partial \rho}{\partial t}+\frac{\partial \rho u_{i}}{\partial x_{i}}=0
$$

Momentum equation:

$$
\frac{\partial \rho u_{i}}{\partial t}+\frac{\partial}{\partial x_{j}}\left(\rho u_{i} u_{j}\right)=-\frac{\partial p}{\partial x_{i}}+\frac{\partial}{\partial x_{j}}\left[\left(\mu+\mu_{t}\right)\left(\frac{\partial u_{i}}{\partial x_{j}}+\frac{\partial u_{j}}{\partial x_{i}}\right)\right] .
$$

$k$ (turbulent kinetic energy) equation:

$$
\frac{\partial \rho k}{\partial t}+\frac{\partial\left(\rho u_{i} k\right)}{\partial x_{i}}=\frac{\partial}{\partial x_{i}}\left[\left(\mu+\frac{\mu_{t}}{\sigma_{k}}\right) \frac{\partial k}{\partial x_{i}}\right]+G_{k}-\rho \varepsilon
$$

$\mathcal{E}$ (dissipation rate of turbulent kinetic energy) equation:

$$
\frac{\partial(\rho \varepsilon)}{\partial t}+\frac{\partial\left(\rho u_{i} \varepsilon\right)}{\partial x_{i}}=\frac{\partial}{\partial x_{i}}\left[\left(\mu+\frac{\mu_{t}}{\sigma_{\varepsilon}}\right) \frac{\partial \varepsilon}{\partial x_{i}}\right]+C_{1 \varepsilon} \rho \frac{\varepsilon}{k} G_{k}-C_{2 \varepsilon} \rho \frac{\varepsilon^{2}}{k},
$$

where $t$ is the time and $\rho$ and $\mu$ are the average density of the volume fraction and molecular viscosity, respectively. $p$ is the pressure, and $G_{k}$ represents the generation of turbulent kinetic energy due to the mean velocity gradients. $\mu_{t}$ is the turbulent viscosity, which can be deduced for turbulence intensity $k$ and energy dissipation rate $\varepsilon$ : 


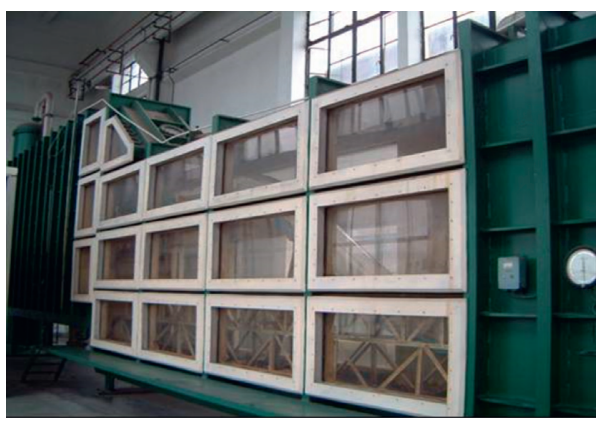

(a)

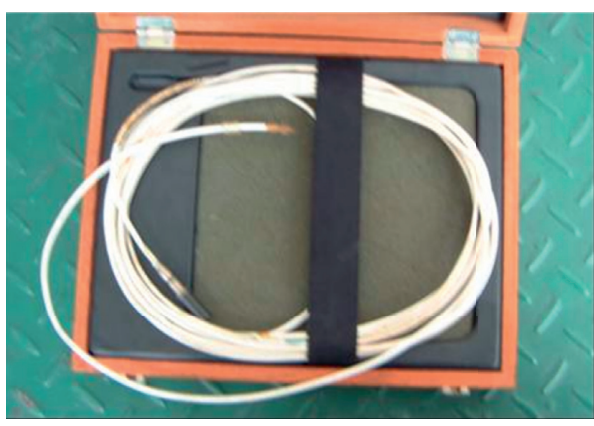

(b)

Figure 3: Related depression test equipment: (a) depression tank; (b) hydrophone.

$$
\begin{aligned}
\mu_{t} & =C_{\mu} \frac{k^{2}}{\varepsilon} \\
C_{1 \varepsilon} & =1.42-\frac{\eta\left(1-\eta / \eta_{0}\right)}{1+\beta \eta^{3}}, \\
\eta & =\frac{S k}{\varepsilon} \\
S & =\sqrt{2 \overline{S_{i j} S_{i j}}},
\end{aligned}
$$

where $S$ is the modulus of the mean strain rate tensor. General constants $\eta_{0}, \beta, C_{\mu}, C_{2 \varepsilon}, \sigma_{k}$, and $\sigma_{\varepsilon}$ of each equation are $4.38,0.012,0.0845,1.68,0.7179$, and 0.7179 , respectively.

The VOF method was introduced to simulate the free surface. The continuity equations of the volume fraction are as follows [22]:

$$
\begin{aligned}
\frac{\partial \alpha_{w}}{\partial t}+u_{i} \frac{\partial \alpha_{w}}{\partial x_{i}} & =0, \\
\rho & =\alpha_{w} \rho_{w}+\left(1-\alpha_{w}\right) \rho_{a}, \\
\mu & =\alpha_{w} \mu_{w}+\left(1-\alpha_{w}\right) \mu_{a},
\end{aligned}
$$

where $u_{i}$ is the velocity components and $x_{i}$ is the coordinates $(i=1,2,3) . j$ is the sum suffix. The air-water interface is tracked by solving the continuity equations. The sum of the water and air volume fraction was one in the controlling body. $\rho_{w}$ and $\rho_{a}$ are the density of water and air, respectively. $\mu_{w}$ and $\mu_{a}$ are the viscosity of water and air, respectively. In the present study, the simulated results will be used for the analysis on hydraulic characteristics of the flow in the pressure tunnel.

The inlet and outlet are both defined as pressure boundaries, where the inlet water level is given by a userdefined function (UDF) and the outlet is given by the boundary condition of the open channel flows. As shown in Figure 5, the grid spacing from the upper horizontal section to plug 1 is $1 \mathrm{~m}$, and the grid spacing is $0.5 \mathrm{~m}$ at the energy dissipation section and lower horizontal section alongside the flow direction. The grid spacing of each section is $0.1 \sim 0.5 \mathrm{~m}$, and the grid spacing of the outlet channel is $0.3 \sim 0.5 \mathrm{~m}$. Except for the unstructured grid with a grid spacing of $0.2 \sim 0.5 \mathrm{~m}$ in each connection part, the rest uses a structured grid.
Figure 6 shows the distribution of the axial velocity of the energy dissipation section in the unusual operational conditions of $h=7.5 \mathrm{~m}$. The inlet position of each plug is squeezed by the water flow and forms a large flow velocity gradient, and a vortex of a certain intensity appears at the entrance angle. The flow of water between the different layers and the vortex area is strongly turbulent. At the end of the plug, due to the sudden enlargement of the cross section, a certain length of the vortex area is formed between the jet and the surrounding water. The jet boundary layer and the vortex area flow vigorously and exhibit turbulent mixing, and they are also the core area of the energy dissipation of the flow caused by the plug energy dissipator.

\section{Results and Discussion}

The grid independence was tested using the grid convergence index (GCI) method, which is a recommended and widely used method for the estimation of uncertainty due to the discretization in computational fluid dynamic (CFD) simulations [23], with grid numbers of 401,562, 562,268, and 755,624 . Figure 7 presents the pressure with different grid numbers and uncertainty of the pressure results on the vertical plug section, where the GCI value is plotted in the form of error bars. From Figure 7, pressure results of the selected three groups of grids are relatively small, and the maximum relative error does not exceed $4 \%$, and the maximum uncertainty was less than $3.2 \%$ (Table 2). Therefore, the number of grids in this study was reasonably set to 562,268.

Figure 8 shows the pressure comparison between experimental and calculated values at the top of the tunnel after plug 3. The maximum relative error in Figure 8 is not more than $13 \%$, and the relatively large error is located in the backflow section after each plug, which is due to the complicated water flow in this area. Good agreement between the experimental and calculated values was obtained, indicating that the numerical simulation results are reliable. With the decrease in $h$, the pressure of the entire lower horizontal section increases, which is beneficial for the antiresistance of the tunnel. Due to the sudden contraction of the flow crosssection before each plug, the flow velocity increases and the pressure decreases. Due to the decreasing inner diameter, the plug pressure is gradually reduced until the outlet position of the plug is suddenly enlarged. The flow 

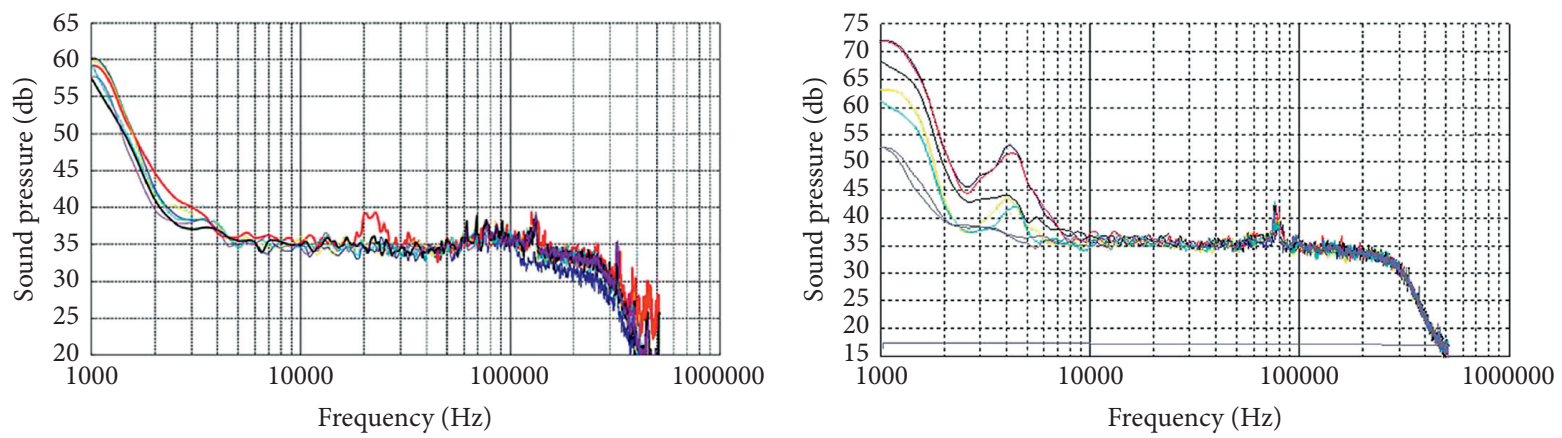

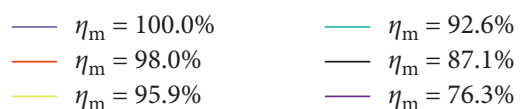

(a)

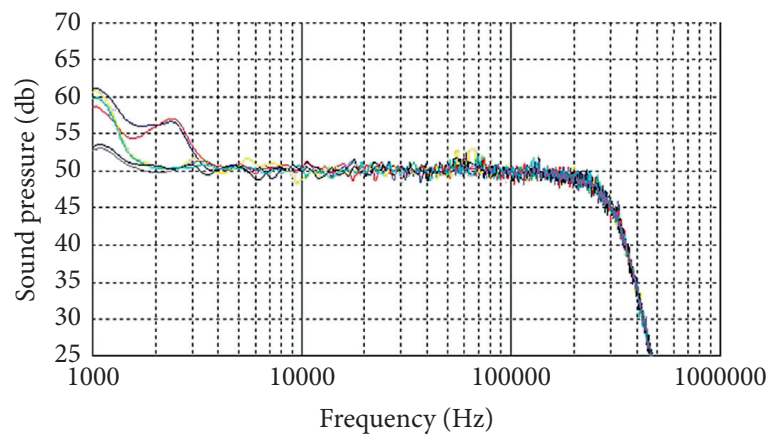

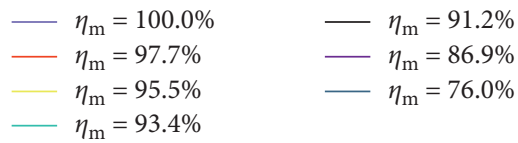

(b)

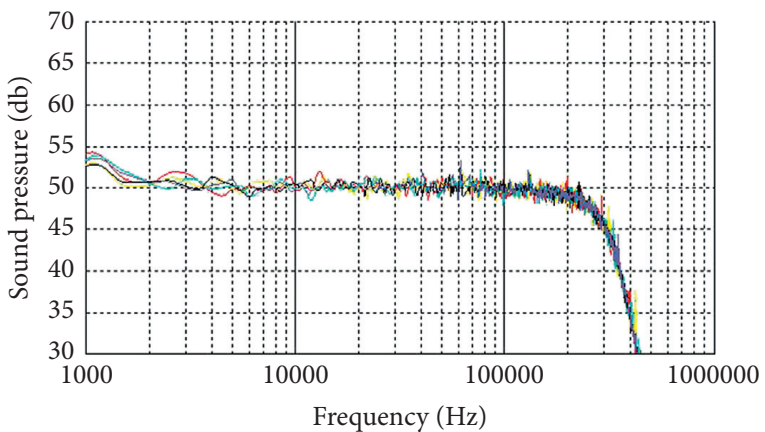

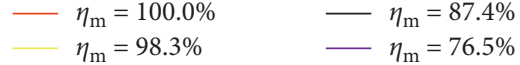

(d)

$\eta_{\mathrm{m}}=96.2 \%$

(c)

Figure 4: Sound pressure signals of each plug: (a) plug 3; (b) plug 4; (c) plug 5; (d) plug 6.

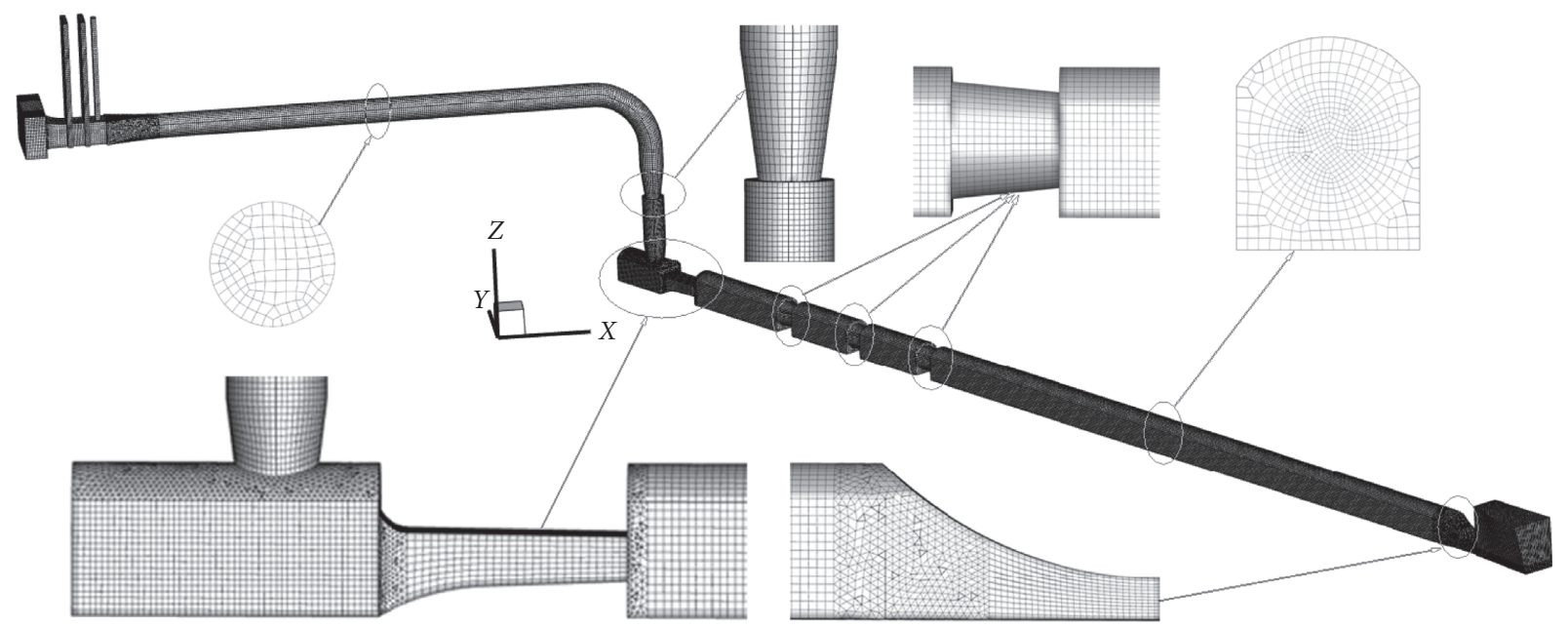

Figure 5: Grid of the calculated model.

forms a recirculating vortex around the jet, and the wall pressure continues to decrease. However, as the flow develops, the pressure begins to rise gradually. The pressure changing trend of each plug is almost the same, but the pressure generally decreases partially after each plug. The pressure tends to be stable after the backflow of the last plug. 


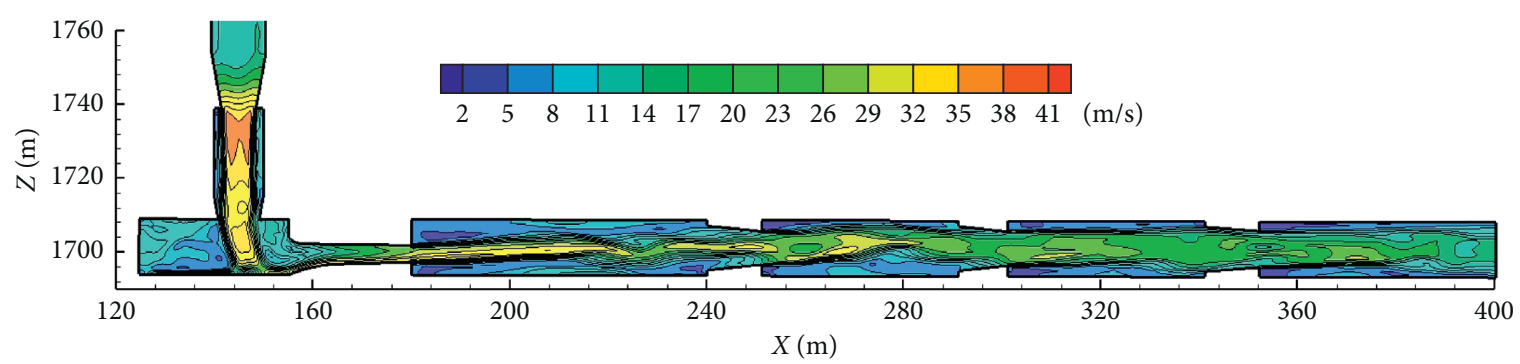

FIGURE 6: Velocity distribution of the energy dissipation section (verification condition, $h=7.5 \mathrm{~m}$ ).
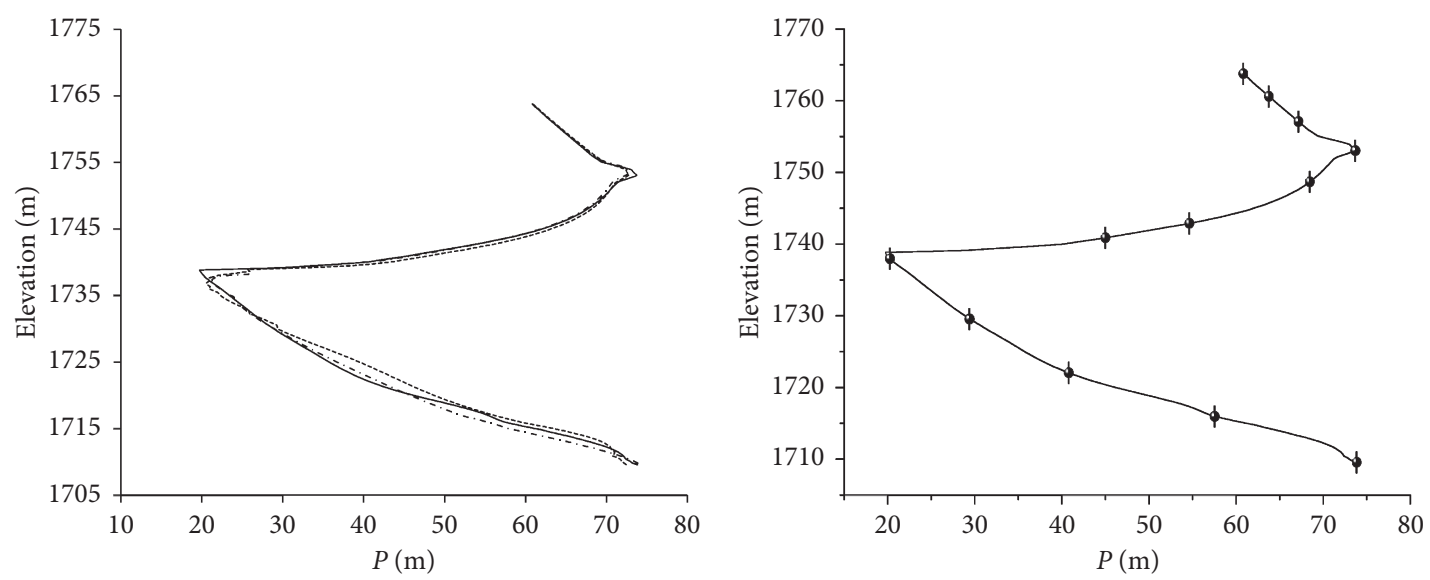

...- Grid numbers of 401,562

— Grid numbers of 562,268

...... Grid numbers of 755,624

(a)

(b)

FiguRe 7: Pressure variation and corresponding uncertainty on the vertical plug section (test number 1): (a) pressure with different grid numbers; (b) pressure variation and corresponding uncertainty.

TABLE 2: GCI value on the vertical plug section.

\begin{tabular}{lccccccccccc}
\hline Elevation $(\mathrm{m})$ & 1763.8 & 1760.6 & 1757.1 & 1753.0 & 1748.7 & 1742.9 & 1738.0 & 1729.6 & 1722.0 & 1715.9 & 1709.6 \\
\hline$P / \mathrm{m}$ & 60.9 & 63.8 & 67.2 & 73.7 & 68.5 & 54.7 & 20.3 & 29.4 & 40.8 & 57.6 & 73.9 \\
GCI (\%) & 2.0 & 2.5 & 1.4 & 0.5 & 1.3 & 3.2 & 0.3 & 0.8 & 1.3 & 1.1 & 0.7 \\
\hline
\end{tabular}

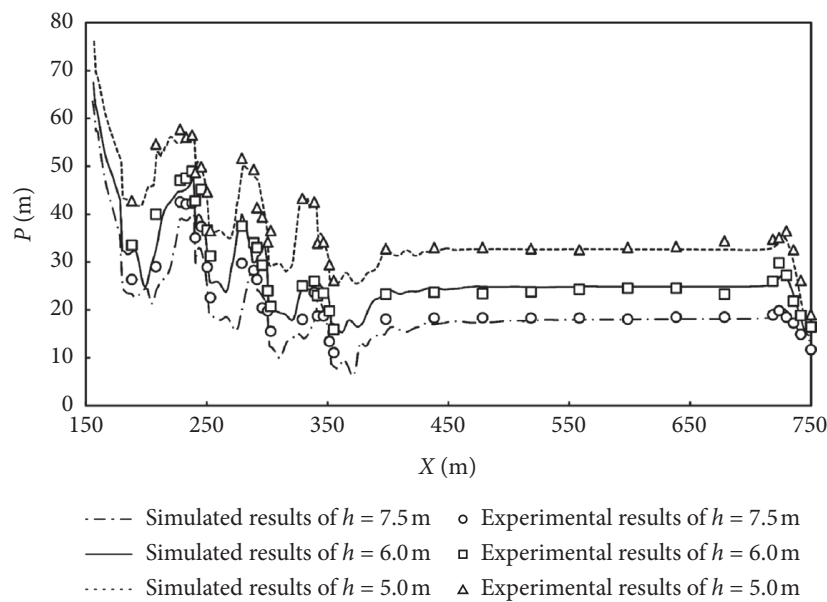

FIgURE 8: Comparison of simulated and tested pressure distributions on the tunnel roof. 
4.1. Discharge Capacity and Discharge Coefficient. The discharge capacity is one of the most important hydraulic factors in discharge structures. This study attempts to enhance the anticavitation ability of plug by reducing the height of the outlet to expand the use of the plug discharge tunnel, but the impact of outlet height on the discharge capacity is a factor that must be considered.

The formula [15] for calculating the discharge capacity of a plug tunnel is

$$
Q=\mu A \sqrt{2 g\left(H_{\text {up }}-H_{\text {down }}\right)}=\frac{1}{\left(\sum \xi_{i}+\lambda(L / D)\right) A \sqrt{2 g\left(H_{\text {up }}-H_{\text {down }}\right)}},
$$

where $A$ is the outlet area, $H_{\text {up }}$ is the reservoir water level, $H_{\text {down }}$ is the downstream water level, $\xi_{i}$ is the local head loss coefficient of each part, $\lambda$ is the resistance loss coefficient along the way, and $L$ is the length of the resistance zone along the way.

The formula (13) shows that when the upstream and downstream water levels are fixed, the discharge is mainly affected by the area of the outlet. In addition, Tong Haihong [24] collected a large amount of model test data and found that the decrease in the outlet height will increase the discharge coefficient of the pressured flood tunnel to a certain extent.

Figure 9 shows the discharge capacity $Q$ and discharge coefficient $\mu$ of the tunnel at different outlet heights. Under the same operational conditions, the discharge capacity does not have a linear relationship with the outlet height. With the decrease in $h$, the discharge capacity gradually decreases and the discharge coefficient gradually increases. At the same outlet height, the discharge coefficient of the tunnel basically does not change with the operational conditions; that is, the discharge coefficient of the plug discharge tunnel is only related to the body shape of the plug and is independent of the operational conditions. Under unusual operational conditions, the area of the outlet was reduced by $46.67 \%$ (from $82.5 \mathrm{~m}^{2}$ to $44 \mathrm{~m}^{2}$ ), and the discharge capacity of the tunnel was reduced by only $13.93 \%$ (from $1393 \mathrm{~m}^{3} / \mathrm{s}$ to $1199 \mathrm{~m}^{3} / \mathrm{s}$ ). This indicates that the influence of the outlet height on the discharge capacity of the plug discharge tunnel is not significant; that is, it is feasible to improve the anticavitation ability of the tunnel by reducing the outlet height. In addition, the discharge capacity of the entire flood discharge system of the hydropower project is generally somewhat larger than the design value.

The outlet height can be determined according to the excess amount of the entire flood discharge system, which means that the discharge reduced by the plug discharge tunnel can discharge through other flood discharge buildings.

4.2. Pressure Characteristics of Tunnel. The pressure of the tunnel roof is shown in Figure 10. It can be concluded that, under the same outlet height condition, the pressure of each part of the flood discharge tunnel is reduced with the decrease in the upstream reservoir water level. This means the normal use of the plug discharge tunnel would

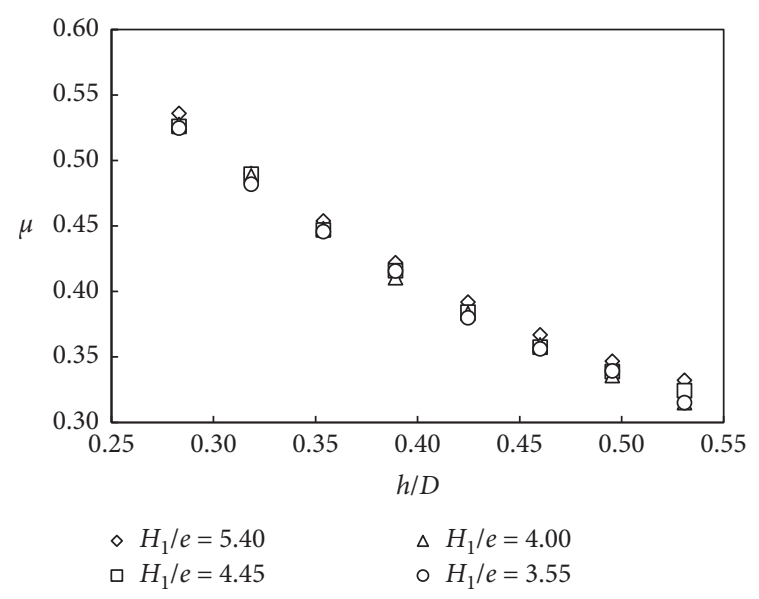

Figure 9: Discharge coefficient of simulation results.

increase the risk of cavitation erosion. The reason for this phenomenon is that, as the reservoir water level decreases, the piezometric head along the tunnel decreases. Under the same operational conditions, with the decrease in the outlet height, the pressure of the plug and subsequent sections increases. This is because as the height of the outlet decreases, under the same upstream and downstream water level conditions, the discharge capacity of the tunnel will decrease, which reduces the flow velocity in the tunnel. According to the energy conservation equation, the pressure on the wall of tunnel increases. However, the change in the pressure of the upper horizontal section $(X=40 \mathrm{~m})$ is relatively low; that is, the change in the pressure of the tunnel caused by the outlet height is mainly concentrated in the plug and its subsequent sections, so the design of the structural strength of the tunnel should focus on the plug and its subsequent sections. In fact, due to the decrease in the outlet height, the reduction in the discharge capacity of the flood discharge tunnel will result in a decrease in the flow velocity in the tunnel. Although the pressure in the upper horizontal section is hardly affected, the cavitation number in the tunnel is increased; that is, reducing the height of the outlet has a positive effect on the anticavitation of the entire flood discharge tunnel.

4.3. Energy Dissipation Rate. The energy dissipation rate is one of the most important parameters of the hydraulic characteristics of energy dissipator, and it is also the most important factor for evaluating the performance of its energy dissipation characteristics. The energy dissipation rate of the plug discharge tunnel can be calculated according to the principle of energy conservation. The energy conservation equation between any two ends of the tunnel is given by the following equation [15]:

$$
z_{i}+\frac{p_{i}}{r}+\frac{v_{i}^{2}}{2 g}=z_{i+1}+\frac{p_{i+1}}{r}+\frac{v_{i+1}^{2}}{2 g}+h_{w}
$$

where $z_{i}, p_{i}$, and $v_{i}$ are the potential energy, average pressure, and average flow velocity of section $i$, respectively, and $h_{w}$ is 


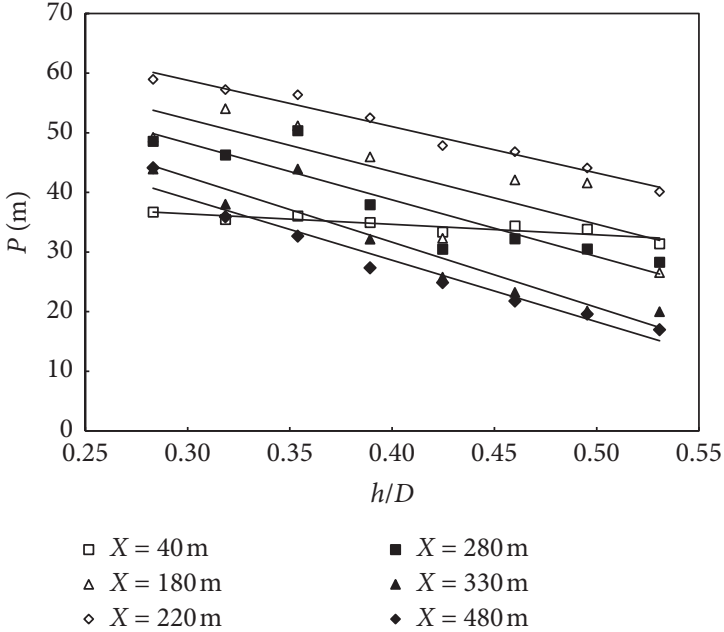

(a)

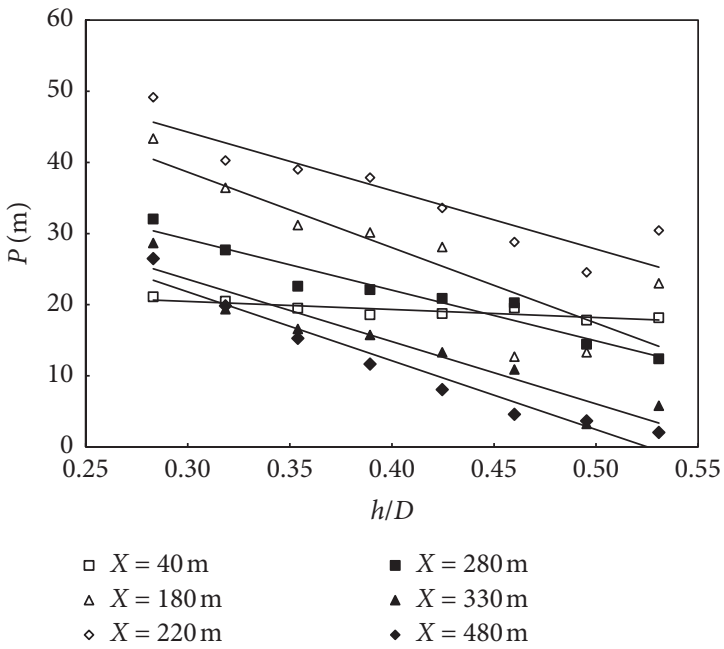

(c)

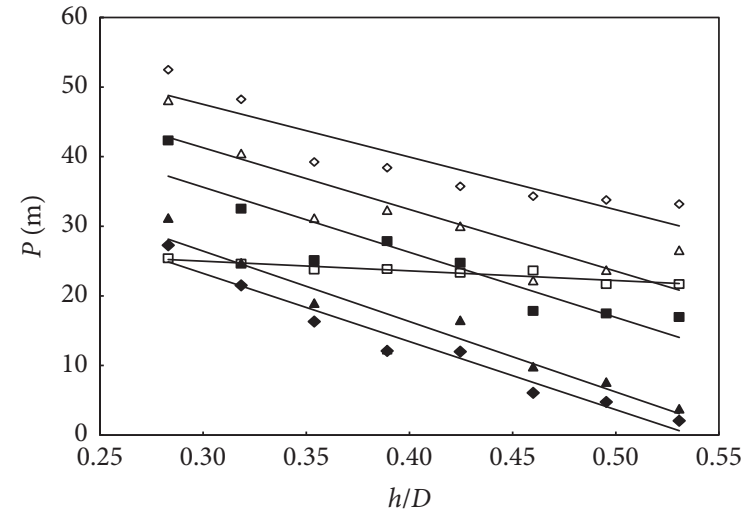
$X=40 \mathrm{~m}$
- $X=280 \mathrm{~m}$
$\triangle X=180 \mathrm{~m}$
$X=330 \mathrm{~m}$
- $X=220 \mathrm{~m}$
- $X=480 \mathrm{~m}$

(b)

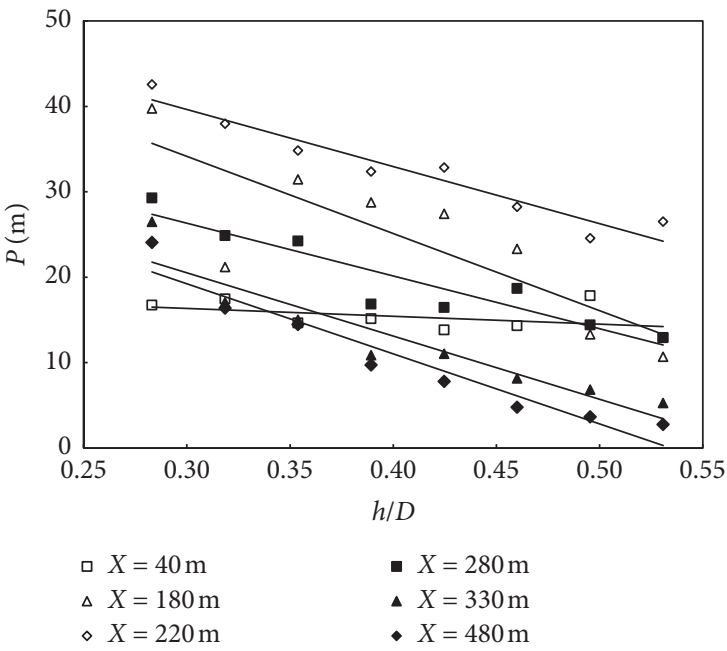

(d)

Figure 10: Pressure distribution of simulation results: (a) $\mathrm{H}_{1} / e=5.40$; (b) $\mathrm{H}_{1} / e=4.45$; (c) $\mathrm{H}_{1} / e=4.00$; (d) $\mathrm{H}_{1} / e=3.55$.

the total head loss between the two sections. We define the energy dissipation rate between any two sections of the plug discharge tunnel as follows [15]:

$$
\eta_{i}=\frac{h_{w}}{H} \times 100,
$$

where $H$ is the water head difference between upstream and downstream and is given by $H=93 \mathrm{~m}+H_{1}-H_{2}$ in this study.

Figure 11 shows the variation in the energy dissipation rate of each plug. The variation rate of the energy dissipation rate of the plugs at different levels is basically the same under different operational conditions. Figure 11(a) shows the variation in the energy dissipation rate in the unusual operational conditions with the change in $h$. Figure 11(b) shows the change in the energy dissipation rate of the whole plug sections with the change in $H_{1} / e$. With the decrease in the outlet height, the energy dissipation rate of the plugs showed a decreasing trend, which was most pronounced for plugs 1-3, while those for plugs 4-6 showed a little change. This is because the total energy dissipation of the system is mainly due to plugs $1-3$, which can also be observed from the turbulent energy distribution shown in Figure 12. The most turbulent area of the entire flood discharge tunnel is the tail area near plugs 1-3. The energy dissipation rate of the entire plug system is also reduced accordingly, and the operational conditions of the flood discharge tunnel have a little effect on the total energy dissipation rate of the plug system; that is, the energy dissipation rate of the plug discharge tunnel is mainly affected by the body shape of the plug. In contrast, the energy dissipation rate is only weakly dependent on the working conditions.

At the end of each plug, due to the suddenly expanding boundary conditions and the inertia of the water flow, the sudden expanding water flow forms a strong turbulence, a strong shear jet, and a large-scale vortex. In the jet boundary layer and the large-scale vortex area, the flow field is turbulent and blended, resulting in significant energy dissipation. Large values of turbulence kinetic energy also appear on the floor and surrounding corners at the end of the \#2 


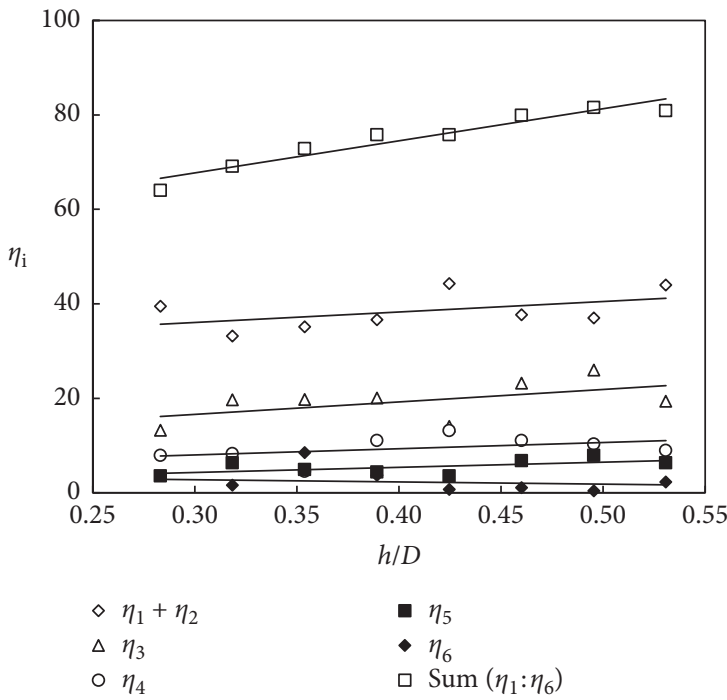

(a)

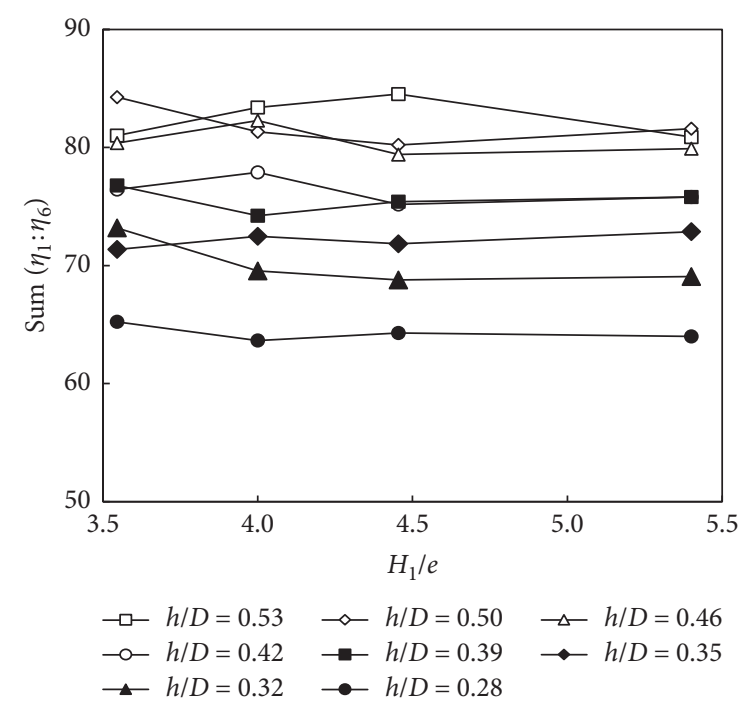

(b)

FIGURE 11: Energy dissipation rate of simulation results: (a) verification condition; (b) total.

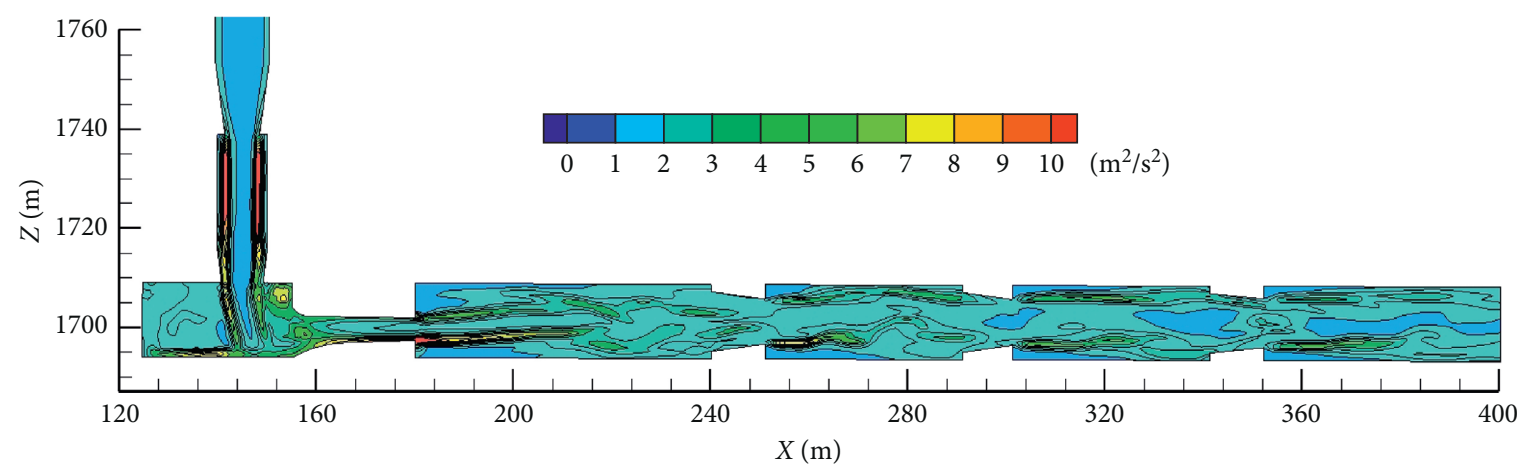

FiguRE 12: Distribution of turbulence kinetic energy of simulation results (verification condition, $h=7.5 \mathrm{~m}$ ).

plug. This is due to the high speed jet from the vertical hole plug impacting the floor. Large-scale vortices, strong turbulence in the impact point, and vortex area cause huge energy loss.

4.4. Cavitation Number. Transient cavitation number [15] can be defined as

$$
\sigma^{\prime}=\frac{\left(p_{u}-p^{\prime}\right)-p_{v}}{\left(\rho v^{2} / 2\right)}=\frac{p_{u}-p_{v}}{\left(\rho v^{2} / 2\right)}-\frac{p^{\prime}}{\left(\rho v^{2} / 2\right)}=\sigma-\frac{p^{\prime}}{\left(\rho v^{2} / 2\right)},
$$

where $p^{\prime}$ is the transient pressure and $\rho$ is the density of water. Based on a large amount of experimental data, Uberoi [25] found that

$$
\sqrt{\overline{p^{\prime 2}}}=0.7 \rho \overline{u^{\prime 2}}
$$

where $u^{\prime}$ is the transient velocity. In the case of Gaussian distribution [15], $p$ equals to $2 \sqrt{\overline{p^{\prime 2}}}$ most of the time, which means

$$
p^{\prime}=2 \sqrt{\overline{p^{\prime 2}}}=2 \times 0.7 \rho \overline{u^{\prime 2}}=1.4 \rho \overline{u^{\prime 2}} .
$$

Then,

$$
\sigma^{\prime}=\sigma-\frac{1.4 \rho \overline{u^{\prime 2}}}{\rho v^{2} / 2}=\sigma-2.8 \frac{\overline{u^{\prime 2}}}{v^{2}}=\sigma-2.8\left(\frac{\sqrt{\overline{u^{\prime 2}}}}{v}\right)^{2},
$$

where $\sqrt{\overline{u^{\prime 2}}} / v$ is the turbulence intensity. According to the predecessor's test results [15], the value of the turbulence intensity near the bottom of highly turbulent flow is close to 0.15 , and then

$$
\sigma^{\prime}=\sigma-2.8 \times(0.15)^{2}=\sigma-0.06 .
$$

It can be seen that the transient cavitation number may be 0.06 lower than the time-averaged cavitation number.

For a plug discharge tunnel that is characterized as operating under extreme conditions, the transition from a submerged state to a free outflow will increase the discharge of the tunnel and increase the flow velocity in the tunnel. The decrease in the reservoir water level reduces the discharge 


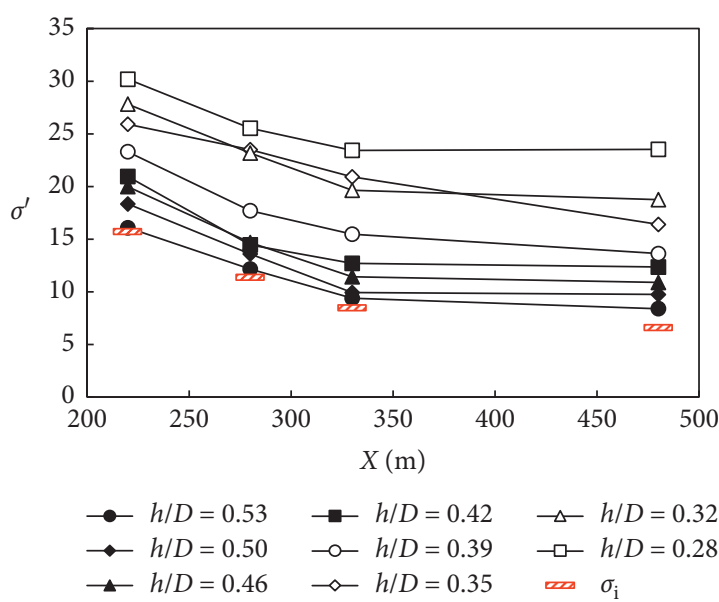

(a)

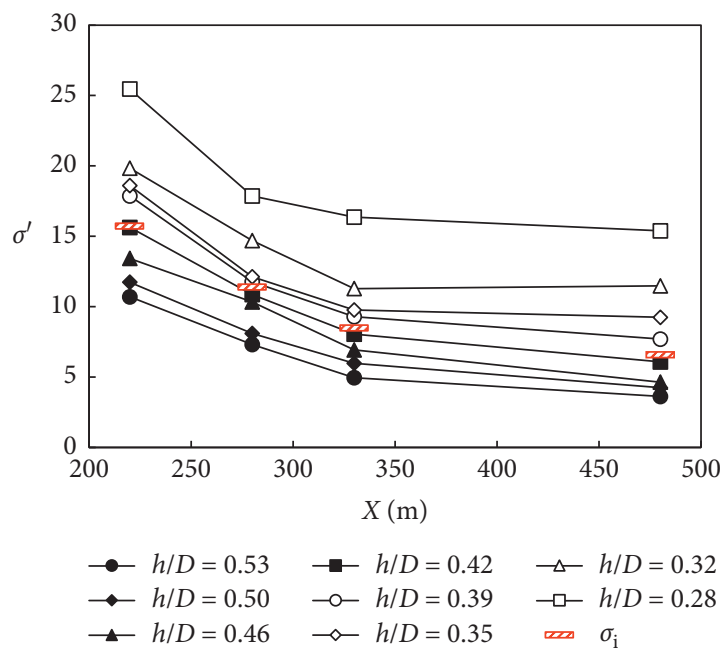

(c)

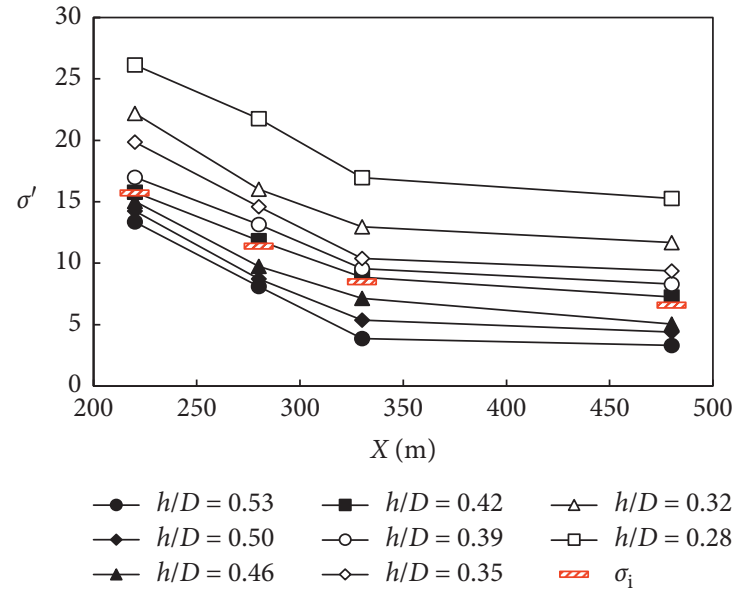

(b)

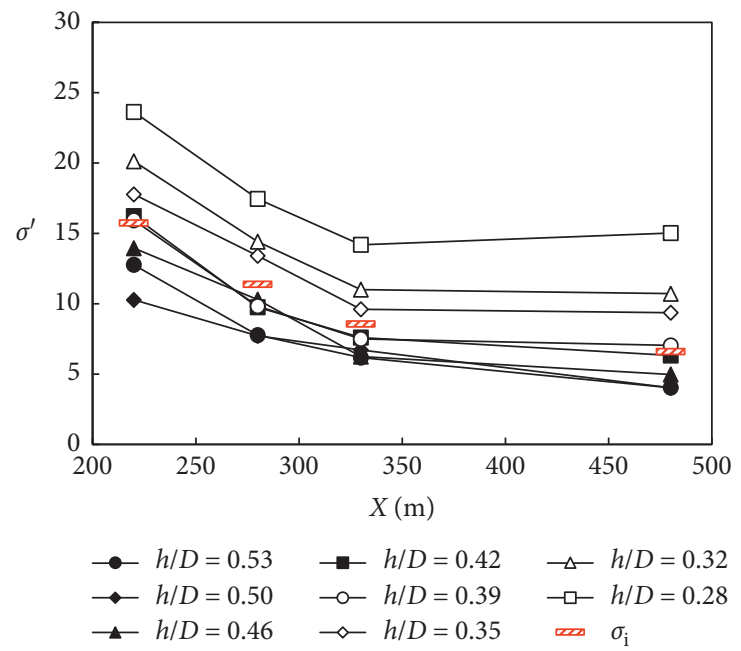

(d)

Figure 13: $\sigma$ : (a) $H_{1} / e=5.40$; (b) $H_{1} / e=4.45$; (c) $H_{1} / e=4.00$; (d) $H_{1} / e=3.55$.

capacity and decreases the wall pressure (Figure 10). These two factors reduce the cavitation number and increase the possibility of cavitation damage in the tunnel. In this study, the outlet is considered as the limiting case; that is, the outlet is kept free. And the change in the cavitation number of different sections is obtained by reducing the water level of the reservoir, thus providing reference information for the normal use of the plug discharge tunnel.

The transient cavitation number in each section is shown in Figure 13 for different operational conditions. Under the same operational conditions, as $h$ decreases, the cavitation number gradually increases. This is because as the height of the outlet decreases, the flow velocity in the tunnel decreases and the pressure increases. According to the definition of the transient cavitation number, it can be known that the transient cavitation number of the flow increases. As the reservoir water level decreases, the decrease in the discharge capacity will result in the decrease in the velocity in the tunnel, while the wall pressure is also reduced. Generally, the transient cavitation number decreases as the reservoir water level decreases; that is, the normal use or reduction in the reservoir water level will increase the risk of cavitation erosion. The reason is that the reduction of the pressure head is lower than that of the velocity head, which results in a decrease in the transient cavitation number. When $h / D$ is less than or equal to 0.53 , the tunnel can meet the requirements of anticavitation under verification conditions. The requirements of antiresistance for $H_{1} / e$ of $4.45,4.00$, and 3.55 , are $h / D \leq 0.42, h / D \leq 0.39$, and $h / D \leq 0.35$ under the normal use conditions, respectively.

\section{Conclusions}

In this study, the normal use of the plug discharge tunnel is realized by changing the outlet height. The key hydraulic parameters are compared using model tests and numerical simulations. The results show the following:

(i) The RNG $k-\varepsilon$ two equation turbulence model can be used to perform numerical simulation of the complex plug discharge tunnel and obtain satisfactory results. 
(ii) The decrease in the outlet height does not significantly reduce the discharge capacity of the plug discharge tunnel. The discharge coefficient is mainly affected by the shape of plug geometry and is irrelevant with the operational conditions.

(iii) The pressure of the upper horizontal section is mainly affected by the upstream reservoir water level.

(iv) The energy dissipation rate of plugs 4-6 is relatively weakly affected by the outlet height, and the decrease in the outlet height mainly reduces the energy dissipation rate of plugs $1-3$, leading to a decrease in the energy dissipation rate of the whole flood discharge tunnel. The energy dissipation rate of the entire flood discharge tunnel is also not related to the operational conditions.

(v) By reducing the height of the outlet, the cavitation number of the flow can be effectively increased, thereby ensuring the safe operation of the plug discharge tunnel. Under the unusual operational conditions, $h / D \leq 0.53$ can meet the requirements of anticavitation. Under the conditions of free outflow, for the $H_{1} / e$ values of $4.45,4.00$, and 3.55 , the conditions in which the tunnel meets the requirements of anticavitation are $h / D \leq 0.42, h / D \leq 0.39$, and $h / D \leq 0.35$, respectively.

\section{Data Availability}

The data used to support the findings of this study are included within the article.

\section{Conflicts of Interest}

The authors declare that they have no conflicts of interests.

\section{Acknowledgments}

This work was supported by the National Science Foundation of China (Grant no. 51939007) and Sichuan Science and Technology Program (Grant no. 2019JDTD0007).

\section{References}

[1] Z. Tian, W.-L. Xu, W. Wang, and S.-J. Liu, "Hydraulic characteristics of plug energy dissipater in flood discharge tunnel," Journal of Hydrodynamics, vol. 21, no. 6, pp. 799-806, 2009.

[2] J.-h. Wu and W.-z. Ai, "Flows through energy dissipaters with sudden reduction and sudden enlargement forms," Journal of Hydrodynamics, vol. 22, no. 3, pp. 360-365, 2010.

[3] W.-z. AI and J.-h. WU, "Comparison on hydraulic characteristics between orifice plate and plug," Journal of Shanghai Jiaotong University, vol. 19, no. 4, pp. 476-480, 2014.

[4] W. Z. Ai and T. M. Ding, "Distance between plugs in multistage plug discharge tunnel," Indian Journal of Geo Marine Sciences, vol. 48, no. 9, pp. 1472-1475, 2019.

[5] W. Z. Ai and T. M. Ding, "Sharp-edged orifice plate's wall pressure characteristics," Indian Journal of Geo Marine Sciences, vol. 48, no. 6, pp. 952-956, 2019.
[6] R. Li, W. L. Xu, J. Luo, H. Yuan, and W. Y. Zhao, "A study on aeration to alleviate cavitation erosion in the contraction section of pressure flow," Journal of Fluids Engineering, vol. 141, no. 9, 2018.

[7] S. O. Russell and J. W. Ball, "Sudden-enlargement energy dissipator for Mica dam," Journal of the Hydraulics Division, vol. 93, no. 4, pp. 41-56, 1967.

[8] X. S. Lin and F. S. Shen, Research and Practice of Multi-Stage Orifice Spill Tunnel, China Water Power Press, Beijing, China, 2003.

[9] J. T. Wang and D. S. Zhang, "Prototype observation experiment of plate dissipator in Xiaolangdi discharge tunnel," China Water Transport, vol. 12, pp. 22-25, 2004.

[10] B. Y. Xiao, "Research on discharge and energy dissipation technology of large-flow and high arch dam in Xiluodu Hydropower Station,” Water Power, vol. 08, pp. 69-71, 2001.

[11] L. J. Lian, W. Wang, and Z. Tian, "Characteristics of energy dissipation and cavitation complex plug in discharge tunnel," Journal of Hydraulic Engineering, vol. 31, no. 2, pp. 62-70, 2012.

[12] L. J. Lian, W. Wang, W. Xu, and Z. Tian, "Numerical simulation of 3-D turbulent flows of plug discharge tunnel," Journal of Sichuan University, vol. 13, no. 3, pp. 1-11, 2011.

[13] S. J. Liu, Y. Q. Yang, W. L. Xu, and W. Wang, "Hydraulic characteristics of throat-type energy dissipator in discharge tunnels," Journal of Hydraulic Engineering, vol. 33, no. 7, pp. 42-46, 2002, in Chinese.

[14] Z. Tian, Study on Hydraulic Characteristics of Plug Energy Dissipator, Sichuan University, Chengdu, China, 2006.

[15] C. G. Wu, Hydraulics, Higher Education Press, Beijing, China, 2008.

[16] J. Deng, W. Xu, J. Zhang, J. Qu, and Y. Yang, "A new type of plunge pool-Multi-horizontal submerged jets," Science in China Series E: Technological Sciences, vol. 51, no. 12, pp. 2128-2141, 2008.

[17] Z. G. Yin, X. W. Cao, J. X. Zhang, and H. D. Shi, "Numerical simulation of the plug discharge under aerated condition," Journal of Hydrodynamics, vol. 22, no. 5, pp. 600-604, 2010.

[18] W. Q. Tao, Numerical Heat Transfer, Xi'an Jiaotong University Press, Xi'an, China, 2nd edition, 2004, in Chinese.

[19] J. Deng, Z. Yang, Z. Tian et al., "A new type of leak-floor flip bucket," Science China Technological Sciences, vol. 59, no. 4, pp. 565-572, 2016.

[20] B. E. Launder and D. B. Spalding, "The numerical computation of turbulent flows," Computer Methods in Applied Mechanics and Engineering, vol. 3, no. 2, pp. 269-289, 1974.

[21] V. Yakhot and S. A. Orszag, "Renormalization group analysis of turbulence. I. basic theory," Journal of Scientific Computing, vol. 1, no. 1, pp. 1-51, 1986.

[22] C. W. Hirt and B. D. Nichols, "Volume of fluid (VOF) method for the dynamics of free boundaries," Journal of Computational Physics, vol. 39, no. 1, pp. 201-225, 1981.

[23] I. B. Celik, U. Ghia, P. J. Roache, and C. J. Freitas, "Procedure for estimation and reporting of uncertainty due to discretization in CFD applications," Journal of Fluids Engineering, vol. 130, no. 7, Article ID 078001, 2008.

[24] H. H. Dong, X. X. Shen, and X. Q. Ding, "Experimental analysis on discharge coefficient of pressure spillway tunnel with contraction outlet," Journal of Yangtze River Scientific Research Institute, vol. 25, no. 4, pp. 97-99, 2008.

[25] M. S. Uberoi, "Correlations involving pressure fluctuations in homogneous turbulence," NACA Tech. note, 3116, 1954. 